\title{
¿Por qué prospera un país? Un análisis cognitivo de las explicaciones en historia*
}

\section{Juan Ignacio Pozo** Mikel Asensio}

Mario Carretero

\section{INTRODUCCION}

En algunos trabajos anteriores hemos hecho referencia a los cambios que se han producido en la última década en los contenidos de la enseñanza de la historia (por ej., Carretero, Pozo y Asensio, 1983; Pozo y Carretero, 1984), que han desplazado éstos desde la simple descripción o narración de los hechos del pasado hasta su explicación en términos de diversos modelos conceptuales. Este paso de lo narrativo a lo explicativo, común por lo demás a otras disciplinas sociales como, por ejemplo, la geografía (Boardman, 1985; Graves, 1982), se justifica por razones exclusivamente científicas, esto es, según la lógica de la disciplina que se trata de enseñar. Sin embargo, el paso de la descripción de un hecho a su explicación -esto es, a responder a preguntas que empiezan con un por qué- trae consigo problemas muy distintos desde el punto de vista del aprendizaje de la historia. Para ofrecer una explicación mínimamente relevante de cualquier hecho histórico se requiere un bagaje de conocimientos y conceptos que no siempre están presentes en la mente de los alumnos que han de aprender esa historia. En ocasiones anteriores hemos insistido en los problemas que tienen los adolescentes para comprender algunos de los sistemas concepruales e instrumentales propios de la historia. Así, el dominio de la cronología histórica no se esta- blece antes de los catorce-quince años (Carretero, Pozo y Asensio, 1983), si bien algunos aspectos del tiempo histórico retrasan mucho más aún su desarrollo (Carretero, Asensio y Pozo, 1985; Pozo, 1985a). Igualmente, los adolescentes tienen serias dificultades en el dominio de las estrategias instrumentales de formulación y comprobación de hipótesis que conducen a la explicación histórica (Pozo y Carretero, 1983). Tienen dificultades también para coordinar dos perspectivas distintas en el análisis o la simple descripción de los hechos históricos (Asensio, Carretero y Pozo, 1986). En suma, carecen de muchos de los requisitos conceptuales necesarios para emprender la ardua tarea de entender - y menos aún elaborar por sí mismos- explicaciones históricas satisfactorias desde un punto de vista científico o disciplinar.

Sin embargo, si bien la calidad de las explicaciones ofrecidas por los adolescentes se ve limitada por todos los factores anteriormente citados, no cabe duda de que los alumnos del ciclo superior de la EGB y de la enseñanza secundaria son capaces de ofrecer explicaciones para ciertos hechos sociales o históricos, aunque éstas no resulten excesivamente sofisticadas. En el presente artículo nos proponemos analizar cuáles son las capacidades explicativas de los adolescentes en el ámbito de la historia. En otras palabras, nos proponemos estudiar es-

* Este trabajo ha sido posible gracias a la concesión de una investigación, dirigida por Mario Carretero, dentro del XIII Plan Nacional de Investigación Educativa. Asimismo, la revisión inicial procede parcialmente de la tesis doctoral de J. I. Pozo dirigida por M. Carretero. La parte empírica ha sido ideada y realizada por M. Asensio.

** Dirección de los autores: Juan Ignacio Pozo y Mikel Asensio, I.C.E. de la Universidad Autónoma de Madrid, 28049 Madrid. Mario Carretero, Facultad de Psicología, Universidad Autónoma de Madrid, 28049 Madrid. 
pecíficamente la naturaleza de las explicaciones históricas adolescentes, tanto en sus contenidos o ideas preferentes como en la estructura o complejidad de las relaciones establecidas.

Para ello será preciso en primer lugar esbozar brevemente los diversos tipos de explicación que suelen aceptarse o proponerse en la historia. Siguiendo una distinción tradicional, y muy fructífera para nuestros propósitos, diferenciaremos entre las explicaciones causales e intencionales de la historia. Dado el carácter psicopedagógico de este trabajo no nos detendremos en la importancia relativa de cada uno de estos tipos de explicaciones dentro de la propia ciencia histórica, sino más bien en los requisitos cognitivos que son necesarios para ofrecer tanto una explicación intencional como causal. Dado que en este mismo número Jesús Domínguez realiza un elaborado análisis de la comprensión "empática» -o explicación intencional-, así como de sus interacciones con las explicaciones causales -o conceptuales-, centraremos nuestros análisis en estas últimas, limitando nuestra exposición con respecto a la explicación intencional a resumir las que, en nuestra opinión, pueden constituir las principales aportaciones de la psicología al estudio de este tipo de explicaciones. Ahora bien, si los adolescentes son capaces de elaborar explicaciones limitadas de ambos tipos, ¿cuándo usarán unas y otras? Intentaremos dar una primera respuesta a esta compleja pregunta, indicando algunos rasgos de las situaciones históricas que hacen más fáciles o más probables uno u otro tipo de explicaciones. Para apoyar los análisis anteriores, presentaremos los datos de una investigación realizada con adolescentes que intenta responder a algunos de los interrogantes planteados. En concreto la investigación se centra en el análisis de las explicaciones que adolescentes de diversas edades ofrecen para una situación histórica determinada (la prosperidad de un país ficticio). Este análisis se centra tanto en los hechos seleccionados por los propios alumnos en sus explicaciones como en la relación de complejidad creciente que establecen entre esos hechos.

\section{TIPOS DE EXPLICACION HISTORICA}

Existen muchos tipos de explicación en la historia y, en general, en las ciencias so- ciales y de la conducta (Borger y Cioffi, 1970; Manninen y Tuomela, 1976; von Wright, 1971). Generalmente suele admitirse una clasificación de las explicaciones históricas en dos tipos: causal e intencional o teleológica. Esta clasificación es también aplicable al resto de las ciencias sociales.

Un hecho se explica causalmente cuando se remite a las circunstancias antecedentes que lo hicieron posible o necesario. Las explicaciones causales miran hacia el pasado y suelen adoptar la forma de una relación condicional suficiente, aunque no siempre sea así. En las ciencias suciales ese pisidus o circunstancias antecedentes que han producido el hecho que queremos estudiar suelen corresponder a una conjunción de factores económicos, sociales, políticos, culturales, etc. Con respecto a cada uno de esos dominios, y a la relación entre ellos, pueden establecerse leyes de mayor o menor generalidad, y de contenido diverso según el modelo del que se parta, que permitirían estudiar de una manera científica el curso de las sociedades.

Las explicaciones causales de los hechos sociales son frecuentes no sólo entre los historiadores sino también en nuestra vida cotidiana. Así, por ejemplo, el día en que redactamos estas líneas, el periódico se abre con la siempre grata noticia de que el Gobierno ha decidido bajar los precios de la gasolina. Este milagroso descenso se explica, según el periódico, por «la mejora en las condiciones del mercado mundial de crudos y el descenso en la cotización del dólar, que es la moneda de pago". Se trata de una explicación claramente causal, en la que ciertas condiciones previas determinan un hecho posterior según ciertas leyes conocidas.

A diferencia de las explicaciones causales, las explicaciones llamadas teleológicas o intencionales no remiten al pasado, sino al futuro. En vez de buscar entre las circunstancias antecedentes de un hecho, intentan más bien indagar en sus consecuencias. Generalmente suelen adoptar la forma de una relación condicional necesaria. Las explicaciones intencionales son frecuentes en la historia ya que los hechos sociales suelen ser acciones humanas motivadas. De esta forma se intentan explicar los hechos históricos no por las causas que los desencadenaron sino por los motivos -es decir, las intenciones con respecto al futuro- de quienes los realizaron. Así, en la misma no- 
ticia de periódico comentada anteriormente el redactor desliza en cierto momento la impresión de que el Gobierno ha decidido la baja de los precios de la gasolina con el fin de respetar los índices de inflación anual previstos, ya que la baja de la gasolina tendrá un efecto reductor sobre el índice de precios al consumo en diciembre. Es ésta una explicación claramente intencional o teleológica, en la que un hecho se explica por las intenciones con respecto al futuro de quienes la ejecutan. Las explicaciones intencionales, a diferencia de las causales, hacen referencia no tanto a estructuras sociales como a motivos psicológicos, ya sean individuales o grupales. Es ésta una diferencia esencial entre ambos tipos de explicación.

En definitiva, podemos resumir las diferencias entre uno y otro tipo de explicación, diciendo que las explicaciones causales se remontan a las estructuras sociales del pasado, mientras las explicaciones intencionales hacen referencia a las consecuencias buscadas por los agentes sociales. En resumidas cuentas las explicaciones causales intentan responder a preguntas que empiezan con un ¿por qué...?, mientras las explicaciones intencionales responden más bien a preguntas finales, que se abren con un ¿para qué...?

El lugar que ha ocupado uno y otro tipo de explicación en la elaboración histórica ha cambiado con el tiempo según el modelo predominante en cada momento. Es éste un debate no sólo disciplinar sino también ideológico en el que no nos compete entrar. Sí tienc, en cambio, interés para nuestros fines el lugar que estas explicaciones han ocupado y ocupan en los libros de texto de historia y ciencias sociales en EGB y BUP.

La vieja historia enciclopédica de reyes y batallas que todos estudiamos era una historia decididamente «heroica», personaliza$\mathrm{da}$, pero además era una historia «con moraleja», en la que todos los hechos y sucesos eran observados, y en su caso explicados, por los grandes fines y metas de la historia, encarnados en héroes legendarios que constituían el auténtico motor de la vida social. Sus motivos eran los grandes motivos de la Humanidad, que habían llegado casi inmaculados hasta nosotros, auténtica «reserva espiritual de Occidente».

Durante los años setenta esa historia se fue sustituyendo por otros contenidos de corte bien distinto. Los hechos pasaron a explicarse por las circunstancias materiales en que fermentaban. Los dibujos de Viriato o El Cid fueron sustituidos por curvas de población, modos de producción y relaciones de poder. Sin embargo, esta historia de modelos estructurales está sufriendo en los últimos años el empuje de los defensores de la «nueva historia», que reclaman un mayor lugar para la «empatía» dentro de la explicación histórica (Dickinson y Lee, 1978; Shemilt, 1984; también Domínguez, en este mismo número). No es casual - y por ello ha sido criticado (Plowright, 1983) - que proyectos tan influyentes en la renovación didáctica de la historia como el de Historia 13/16 (Varios, 1985) se abran con una unidad didáctica basada en un caso de detectives.

Sin embargo, la recuperación de la explicación intencional que se reclama no supone una vuelta a la vieja historia sino, entendemos, un intento de integrar causas $\mathrm{e}$ intenciones en un modelo común, propuesta de integración llena de matices y dificultades que quedan perfectamente reflejados en el trabajo de Jesús Domínguez que acompaña a éste. De hecho, como muestra la noticia referida anteriormente, ambos tipos de explicación no resultan excluyentes sino más bien complementarios.

Con todo, en la mayor parte de los libros de texto aún vigentes lo que se encuentra, más que una diferenciación como la que aquí venimos sosteniendo, es una notable confusión - que no integración-entre diversas formas de explicación. Al alumno no se le presentan diversos modelos explicativos sino más bien un cóctel de explicaciones, en el que indiscriminadamente unos hechos son explicados de una forma y otros de otra, sin que el alumno llegue a conocer la diferencia entre ambas. Esta indiferenciación es en nuestra opinión contraria a los propios objetivos de la enseñanza de la historia. Pero además conlleva un peligro adicional, ya que los procesos psicológicos que hay que poner en marcha para alcanzar o comprender la explicación son distintos en cada caso. No hay razones de principio para creer que los alumnos tengan que dominar por igual ambos tipos de explicación, ya que cada uno conlleva problemas o sesgos específicos que es necesario superar. Resulta conveniente, por tanto, hacer un análisis, meramente orientativo, de los requisitos 
psicológicos necesarios para emprender una explicación causal o intencional en historia.

\section{LA EXPLICACION CAUSAL EN HISTORIA}

El estudio de la causalidad ha ocupado a las mentes más lúcidas de la Humanidad desde tiempos muy remotos. Los debates y las discusiones en torno a este problema son, por tanto, interminables, por lo que en esta ocasión nos vemos obligados a eludir$\operatorname{los}^{6}$. Nos limitaremos a presentar los rasgos generales de toda explicación causal para, a continuación, señalar algunas de las peculiaridades de la causalidad histórica.

Desde nuestro punto de vista (desarrollado con amplitud en Pozo, 1985b) en las explicaciones causales pueden distinguirse tres componentes psicológicos, de diverso interés para la educación: principios, teorías y reglas de inferencia. En primer lugar, existirían unos principios o leyes generales de la causalidad. Estos principios determinarían la forma que adopta toda explicación causal, sea cual sea su contenido o complejidad. Así, a partir de Bunge (1959) creemos que toda relación causal es:

- Constante, esto es, a igualdad de circunstancias, la misma causa se sigue siempre de los mismos efectos. En otras palabras, todas las relaciones causales responden a leyes más o menos complejas.

- Asimétrica, ya que la causa debe preceder o, como máximo, ser simultánea al efecto. Esta dimensión temporal de la causalidad adquiere dimensiones propias en el ámbito histórico, marcadas por la duración diversa de los intervalos causales.

- Condicional, puesto que la causa es una condición para la aparición del efecto. La relación condicional puede ser necesaria, suficiente, necesaria y suficiente, o simplemente contribuyente.

- Productiva, ya que la causa no se limita a preceder constantemente al efecto, sino que es su origen, esto es, lo genera o produce.

Hay indicios suficientes (véase Bullock, Gelman y Baillargeon, 1982; Pozo, 1985b) para creer que estas leyes generales de la causalidad se dominan desde edades muy tempranas, aunque no se sea consciente de ellas, por lo que su interés educativo es muy secundario. Mayor interés tienen los otros dos componentes del pensamiento causal.

Además de poseer una forma general, recogida en los principios, las explicaciones causales tienen un contenido propio. Así, creemos que un determinado virus es la causa de cierta enfermedad o que un accidente de tráfico con graves consecuencias se produjo a causa de un exceso de velocidad. Igualmente, en la historia diversos modelos sostienen la existencia de una relación causal entre ciertos tipos de hechos. A diferencia de los principios, que son los mismos en todas las relaciones causales, las ideas o teorías sobre lo que está relacionado causalmente pueden variar considerablemente de una persona a otra. Esto es especialmente cierto en el caso de las ciencias sociales, en las que toda explicación es relativa al marco teórico y los datos en que se apoya (Asensio, Carretero y Pozo, 1986). De hecho, frecuentemente existen diversas teorías para explicar un mismo acontecimiento histórico. Como señala Fischhoff (1980), la debilidad de las teorías históricas se pone de manifiesto en su incapacidad para predecir hechos futuros, que en definitiva muestra la inseguridad de sus explicaciones con respecto al pasado.

Pero a pesar de su debilidad las teorías causales son imprescindibles para comprender el mundo que nos rodea (Pozo, 1985b). Podemos decir sin titubear que la calidad y eficacia de las explicaciones causales depende de las teorías en que se apoyan. Y en este sentido los adolescentes se hallan claramente desfavorecidos, ya que, como veremos más adelante, sus ideas causales en historia son relativamente pobres y están poco relacionadas entre sí. Por todo ello, parece claro que uno de los objetivos fundamentales de la enseñanza de la historia debe ser dotar a los alumnos de teorías o modelos sobre el funcionamiento y cambio de la sociedad, esto es, de marcos conceptuales útiles para entender la realidad social e histórica (sobre el desarrollo de los conceptos históricos véase Carretero, Pozo y Asensio, 1983; Pozo y Carretero, 1984; una clasificación semejante aplicada a la geografía puede encontrarse en Naish, 1982).

Pero el saber histórico no sólo consta de ideas sino también de reglas de inferencia 
que permiten decidir qué explicaciones son las más adecuadas en cada momento. $\mathrm{A}$ pesar de su naturaleza relativa, no cabe duda de que las explicaciones pueden y deben contrastarse con los datos disponibles. Para ello es necesario poseer un conjunto de reglas instrumentales o metódicas. Ese conjunto de reglas forman parte del método del historiador. En los últimos años, al calor de algunos movimientos renovadores en la enseñanza de la historia (para una revisión véase Pozo, Carretero y Asensio, 1983), se viene insistiendo cada vez más en la importancia de que el alumno domine las técnicas metódicas del historiador con el fin de llegar a elaborar por sí mismo explicaciones de los hechos históricos. Se trata de que el alumno haga historia en lugar de recibirla ya hecha. Para ello es sin duda necesario que domine el pensamiento hipotético-deductivo en historia (Pozo y Carretero, 1983) y que maneje con cierta fluidez los conceptos metódicos de la historia (fuente, prueba, causa, etc.). Ahora bien, si estas habilidades, vinculadas al desarrollo del pensamiento formal, son necesarias para alcanzar esos propósitos, no parece que sean suficientes (Carretero, 1985; Pozo y Carretero, 1986). Como señalábamos anteriormente, es imprescindible poseer ideas causales eficaces. De lo contrario, el alumno sabrá comprobar adecuadamente las explicaciones que se le propongan, pero no será capaz de elaborar por sí mismo explicaciones adecuadas. Por ello creemos que si se quiere enseñar realmente al alumno a hacer historia es necesario no sólo proporcionarle los métodos o reglas de inferencia del historiador, sino también en buena medida las ideas o teorías del historiador.

Pero además de estos rasgos generales, compartidos con el conocimiento causal en otras disciplinas, la causalidad histórica tiene algunas características peculiares. Aquí quisiéramos resaltar sólo dos que tienen una especial importancia.

En primer lugar, el intervalo temporal entre causa y efecto, al que aludíamos anteriormente, es en historia especialmente grande. Mientras que en la causalidad física suele ser aplicable una regla de «contigüidad temporal» entre causa y efecto, en historia es frecuente que los hechos tengan consecuencias no sólo a corto sino también a largo plazo. Por ejemplo, las causas de la situación política en Oriente Próximo se remontan a muchos años, incluso siglos, atrás.
Esto plantea un problema adicional en la comprensión de la çausalidad histórica no presente en otras disciplinas. Los adolescentes tienen serias dificultades para remontarse en busca de causas o consecuencias distantes en el tiempo, no sólo por los problemas de tiempo histórico que esto plantea (Carretero, Pozo y Asensio, 1983; Pozo, 1985a), sino también porque establecer relaciones causales a largo plazo requiere realizar cadenas causales con un número de eslabones cada vez mayor (Adelson, 1971), lo que implica hacer operaciones mentales sobre operaciones previamente realizadas, uno de los rasgos que definen al pensamiento formal (Carretero, 1985).

Un segundo rasgo característico de la explicación causal en historia es que muy rarameıte los hechos históricos poseen una sola causa y tienen una sola consecuencia. Por tanto, las relaciones causales en historia no son nunca simples o lineales, sino siempre complejas, requiriendo la integración de varios factores en una explicación común. Más adelante, en el trabajo empírico, volveremos sobre este problema, mostrando las dificultades que tienen los adolescentes para establecer redes causales complejas en historia.

\section{LA EXPLICACION INTENCIONAL EN HISTORIA}

La aportación de la psicología a este tipo de explicaciones no se limita, como en el caso de la causalidad, a analizar su desarrollo en los alumnos. Dado que las explicaciones intencionales se basan en la atribución de motivos a las acciones de personas concretas, sea individual o colectivamente, tienen en última instancia una naturaleza psicológica. En otras palabras, para realizar explicaciones teleológicas de tipo intencional se requiere cierto tipo de conocimiento psicológico. Esta afirmación es válida tanto para el historiador como para el alumno de historia. Por ello la aportación de la psicología adquiere en este caso una dimensión especial.

Entre los historiadores que sitúan en un lugar central la explicación intencional están aquellos que consideran que la historia es, ante todo, el estudio de las «ideas» o de las «mentalidades» a través del tiempo. Así, por ejemplo, tanto Collingwood (1946) como Febvre (1953) especulan sobre la fun- 
ción que debe desempeñar la psicología en el análisis de la historia. Ambos son conscientes de que si los hechos históricos se deben, al menos en parte, a las intenciones de sus agentes, es necesario conocer la mente de éstos para poder entender plenamente la historia. Sin embargo, ambos coinciden en rechazar el papel que pudiera desempeñar la psicología en el conocimiento de esas mentes del pasado, ya que, según ellos, la psicología contemporánea es incapaz de captar la esencia de las mentalidades de los hombres de otros tiempos. Por ello, acaban proponiendo, de una u otra forma, un modelo en el que la mente sería una «caja negra» sobre la que poco o nada sabemos, y con respecto a la cual lo más que podemos es «aducir» argumentos (Booth, 1980; Shemilt, 1984). Este modelo de «caja negra», que tan familiar nos resulta a los psicólogos tras varias décadas de rigores positivistas en psicología, supone una renuncia al conocimiento científico de las mentalidades de otros periodos.

Sin embargo, estas argumentaciones son, en nuestra opinión, escasamente consistentes. La psicología puede y debe desempeñar una labor eficaz en la comprensión de las mentes del pasado. En primer lugar, aunque esas mentes tengan una naturaleza histórica y cambien con el tiempo, hay que aceptar la existencia de ciertos rasgos humanos estables por encima del tiempo (Bizière, 1983). Pero incluso en aquellos rasgos cambiantes con el tiempo es posible un análisis científico, desde la psicología, de esa evolución. De hecho, ése es el propósito de ciertas tendencias de psicología dialéctica (por ej., Riegel, 1976, 1977), cuyo objetivo principal es precisamente estudiar la evolución de los «modelos de mundo» que poseen las personas como consecuencias de los cambios sociales producidos en el ambiente en que se mueven esas personas (con respecto al análisis de los modelos de mundo en la psicología actual véase Zaccagnini, 1985).

Pero además de ayudarnos a comprender mejor la naturaleza de las explicaciones intencionales en historia, la investigación psicológica puede ayudarnos a conocer mejor los procesos psicológicos necesarios para emprender o comprender este tipo de explicaciones.

Para comprender una explicación histórica intencional hay que ser capaz de atri- buir correctamente motivos a las acciones de personas o colectividades, generalmente distantes en el tiempo.

Diversas investigaciones psicológicas han mostrado que la capacidad de atribuir motivos a las acciones de los demás se desarrolla con la edad. Así, por ejemplo, Adelson (1971), en una sugestiva investigación, observó un claro desarrollo de esta habilidad durante la adolescencia. Por ejemplo, si se preguntan las razones por las que una persona puede reincidir en el delito y volver a la cárcel, puede observarse una notable diferencia cualitativa entre las respuesras dadas por los adolescentes más jóvenes (doce-trece años) y los mayores (quince-diecisiete). Los primeros responden frecuentemente de un modo tautológico: «porque es un criminal» (Adelson, 1971, pág. 1018), manifestando una absoluta incomprensión de los motivos que subyacen a las acciones de las personas, en este caso de los que puede tener una persona para cometer un delito. Manifiestan también incapacidad para descentrarse de su propia situación, desde la que juzgan las acciones de los demás: (vuelven a la cárcel...) «porque no conocen otra cosa y tienes que darles una lección» (ibíd. pág. 1019). Por el contrario, los adolescentes de quince años en adelante dan respuestas más elaboradas, en las que se muestra un intento de explicar las acciones de la gente a partir de motivos subyacentes: "porque ir a la cárcel produce envidia hacia los demás» ... "porque se vuelven amargados o se sienten burlados» (ibíd., pág. 1019).

Adelson comprueba también que hasta bien avanzada la adolescencia no se comprenden los motivos de las acciones que emprende toda una comunidad, mientras que la comprensión de motivos individuales resulta más fácil (Adelson y O'Neil, 1966). Esto es debido a la incapacidad del joven adolescente para imaginarse una colectividad abstracta, lo que le lleva a personalizar las instituciones sociales y a interpretar las acciones comunitarias en función de motivaciones meramente individuales (Adelson, 1971).

Pero la comprensión de las explicaciones intencionales en historia no precisa sólo de una descentración en el espacio, sino también en el tiempo, como señala Febvre (1953, pág. 40 de la trad. cast.) en su definición de historia: 
«Es el estudio científicamente elaborado de las diversas actividades y de las diversas creaciones de los hombres de otros tiempos, captadas en su fecha, en el marco de sociedades extremadamente variadas y, sin embargo, comparables unas a las otras:»

Hallam (1972) considera que uno de los mayores impedimentos que tienen los adolescentes para comprender situaciones históricas y razonar adecuadamente sobre ellas es su tendencia a juzgar cualquier hecho histórico desde la perspectiva del siglo XX. Luc (1979) señala las dificultades que tienen los niños para manejar documentos históricos debido a ciertas tendencias «egocéntricas y finalistas» (ibíd., pág. 69 de la trad. cast.) que les impiden situar esos documentos en su momento histórico preciso.

Este egocentrismo es particularmente difícil de superar, ya que se requiere entender las diferencias entre las mentalidades de otros tiempos y «el modelo del mundo» actual, que es el que aplican diariamente los adolescentes a su vida. El lento progreso en la superación de este «sesgo de disponibilidad» (Tversky y Kahneman, 1974) queda perfectamente reflejado en los análisis de Domínguez (en este mismo número; también Shemilt, 1984). Los adolescentes, que difícilmente comprenden el cambio social (Shemilt, 1980), encuentran aún mayores problemas para comprender el cambio «mental». No obstante, estos problemas, como muestra el propio J. Domínguez, no son insensibles a la instrucción. Además, su superación se halla estrechamente ligada al progreso de las explicaciones causales.

En este punto, en que volvemos a la relación entre ambos tipos de explicación, una vez analizados sus rasgos psicológicos fundamentales, estamos en condiciones de preguntarnos por las circunstancias en que el adolescente hace uso de las diversas formas de explicación histórica. ¿Cuándo utiliza el adolescente la explicación causal y cuándo la intencional?

\section{EXPLICACION CAUSAL E INTENCIONAL EN LOS ADOLESCENTES}

La atribución de un efecto a factores internos -o intencionales - y. externos -o causales $-{ }^{7}$ ha sido estudiada con intensi- dad por los psicólogos sociales. Así, Harold H. Kelley $(1967,1973)$ formuló la «teoría de la atribución» cuyo propósito fundamental es determinar cuándo las personas atribuimos la ocurrencia de un hecho social a las motivaciones de las personas que lo ejecutan $o$ a las circunstancias externas en que tiene lugar. Es por tanto, una teoría -aunque no se reconozca explícitamente- sobre las situaciones en que usamos explicaciones intencionales o causales (Buss, 1978). Kelley propone que para decidir entre una y otra, «el hombre de la calle, el psicólogo [o historiador, añadimos nosotros] ingenuo, utiliza una versión ingenua del método usado en la ciencia» (Kelley, 1973, pág. 109). Este método según Kelley consistiría en un análisis de las situaciones de acuerdo con tres criterios:

- Consistencia: un factor es seguido consistentemente por el efecto. Por ej., siempre que hay una baja en el precio del petróleo, el gobierno baja la gasolina.

- Discriminabilidad: otros factores potenciales no son seguidos sistemáticamente por el efecto. Por ej., otros años, la inflación ha superado las previsiones sin que el gobierno bajara la gasolina.

- Consenso: en otras personas o agentes se produce el mismo efecto ante el mismo factor. En las mismas circunstancias, casi todos los gobiernos de Europa han bajado el precio de la gasolina.

Siempre que se cumplen estas tres condiciones el efecto será atribuido a una causa externa, esto es, será explicado causalmente. En cambio, cuando no se cumplan se atribuirá más bien a las intenciones idiosincráticas del agente.

A pesar de su indudable interés y de la gran cantidad de investigaciones que se han realizado a partir de esta teoría (véase Kelley y Michella, 1980), el modelo normativo, de "científico ingenuo», postulado por Kelley queda en evidencia ante la gran cantidad de estudios que muestran que las personas suelen realizar atribuciones sesgadas cuando se enfrentan a problemas sociales (Nisbett y Ross, 1980). Lejos de utilizar un método riguroso y sistemático para decidir qué factores determinan la aparición de un hecho, tanto los adolescentes como los adultos tienden a guiarse por información su- 
perficial, a veces irrelevante. Podemos decir que la mayor parte de las personas aplican un tipo u otro de explicación en función de unas reglas simplificadoras -o beurísticos- que toman unos pocos indicios de la situación como criterio de decisión en lugar de hacer un análisis sistemático y exhaustivo de la misma, como suponía Kelley $(1967,1973)$. A continuación vamos a referirnos brevemente a algunos de estos sesgos, desarrollados de modo más exhaustivo en otros lugares (Carretero y García Madruga, 1984; Kahneman, Slovic y Tversky, 1982; Nisbett y Ross, 1980; Pozo, 1985b; de Vega, 1984).

Para decidir si un hecho debe explicarse preferentemente de un modo causal o intencional, el primer criterio simplificador que suele usarse es una regla de semejanza entre antecedente y consecuente, por la que ambos deberían tener una naturaleza común. Esta regla de semejanza denominada también «heurístico de representatividad» (Tversky y Kahneman, 1974) es la que nos hace creer, por ejemplo, que los trastornos emocionales en las personas tienen causas igualmente emocionales o que «a grandes males, grandes remedios». A plicada a la historia, esta regla supone que los fenómenos con una dimensión social, económica o política tienen causas de naturaleza también social, económica o política. Como veremos más adelante esta concepción, sin duda errónea, es especialmente importante en el caso de los adolescentes, habida cuenta de la desconexión que para ellos existe entre esos dominios. Pero la regla de semejanza puede desembocar también en una explicación intencional. Cuando el hecho estudiado tenga rasgos de acción individual tenderá a proponerse una explicación intencional. Así suelen explicar los adolescentes, por ejemplo, los viajes de Colón o la ascensión de Hitler al poder en Alemania.

Pero la tendencia a atribuir de modo exclusivo las acciones a los agentes tiene su apoyo además en otro sesgo que, dentro de la teoría de la atribución, ha recibido el significativo nombre de error fundamental de atribución (Ross, 1977; Nisbett y Ross, 1980 ). Se ha comprobado que en una misma situación el agente y un observador realizan atribuciones distintas. Se ha comprobado que quienes ejecutan una acción suelen atribuirla a las presiones o circunstancias externas que les inducen a hacerla, mientras que el observador la atribuye a las propias intenciones o a la personalidad del agente. A esta tendencia del observador a proponer explicaciones intencionales se le denomina error fundamental de atribución. Es obvio que en la historia que normalmente se presenta en el aula, el alumno no pasa de ser un observador ajeno a la propia situación y por tanto es posible que tienda a dar, con más frecuencia de la deseada, explicaciones personalizadas de la historia. Curiosamente, aquellas situaciones didácticas -como, por ej., dramatizaciones o juegos de simulación (Martín, 1983; Piñeiro y Gil, 1984) - que hagan del alumno un protagonista de la historia en un contexto realista le ayudarán a superar ese error.

Hacer historia es una forma de comprender la naturaleza plural y compleja de las explicaciones históricas, ya que hace posible que el alumno conozca y sienta otras realidades -y mentalidades- distintas de la suya. De esta forma será más fácil que supere el sesgo de disponibilidad (Nisbett y Ross, 1980; Tversky y Kahneman, 1974) en las explicaciones históricas, al que aludíamos unas páginas más atrás. Según este sesgo, una explicación sería más plausible cuanto más disponible -o fácil de recuperar- estuviera en la memoria, y sería más disponible cuanto mayor fuera la frecuencia con que se usa. Así, como señala Domínguez (en este mismo número), los adolescentes parten de la realidad disponible actualmente para explicar retrospectivamente el pasado. Para vencer esta tendencia es necesario aumentar la disponibilidad de otras realidades o mentalidades, y ello difícilmente será posible mediante el simple recurso al libro de texto. Es preciso un esfuerzo de imaginación que haga la historia más viva y menos «libresca» (Luc, 1979).

En este sentido son muchas las investigaciones que muestran que cuanto más concreta es una información mayor es su impacto sobre las ideas de quien la recibe (Nisbett y Ross, 1980). Así, podemos escuchar cada lunes pavorosas estadísticas sobre el número de accidentes y muertos en la carretera el fin de semana, sin inmutarnos lo más mínimo. Pero basta que pasemos por un lugar donde acaba de producirse un grave accidente y veamos sus consecuencias para que sintamos los accidentes como un peligro muy real y levantemos el pie del acelerador. De la misma forma, en la clase de historia puede ser muchas veces más útil presentar información concreta bien selec- 
cionada, que sirva como ejemplo o modelo de los rasgos o conceptos más abstractos que se pretende enseñar, en vez de presentar directamente estos últimos.

En definitiva, todos estos sesgos, y algunos otros que aún podríamos presentar - como, por ejemplo, la mayor disponibilidad psicológica del futuro con respecto al pasado (Pozo, 1985a) o la influencia del formato en que se presenta la informaciónno son sino tendencias cognitivas presentes no sólo en el adolescente sino también en el adulto no especializado. Estas tendencias determinan el tipo de comprensión y/o explicación que se obtendrá a partir de la información disponible. Los profesores de historia y ciencias sociales han de ser conscientes de que para que sus alumnos alcancen un mejor nivel en sus explicaciones históricas, en el que logren integrar los factores causales e intencionales, deben remontar estos sesgos. Su alumnos procesan la información histórica de un modo selectivo. ¿Mostrarán preferencia en sus explicaciones por unos hechos en lugar de otros? ¿Qué dominios de la realidad social - cultural, económico, político- les parecen más relevantes para explicar los hechos históricos? ¿Hay un predominio durante la adolescencia de un tipo de explicación con respecto a otro? ¿Qué grado de integración hay entre los diversos factores causales e intencionales que conocen? Con objeto de ofrecer una primera respuesta a estas preguntas hemos emprendido una investigación que se describe a continuación.

\section{ESTUDIO EXPERIMENTAL}

\section{Introducción}

Hasta el momento hemos visto que existen diferentes tipos de explicaciones. Fundamentalmente hemos distinguido entre la explicación causal y la explicación teleológica. Y hemos sostenido que ambas explicaciones requieren unos procesos psicológicos diferentes. A partir de estos planteamientos nos interesamos por el tipo de explicación que se recogía en las asignaturas de ciencias sociales en los distintos niveles educativos. Más concretamente, nos interesamos por estudiar cuáles eran los factores y los tipos de factores que los alumnos eran capaces de manejar como explicativos de una determinada situación. Y también pretendíamos estudiar cuáles son las configuraciones de relaciones que los alumnos de diferentes niveles establecerán entre los distintos factores explicativos.

En una investigación anterior (Carretero, Pozo, Asensio, 1983) habíamos observado que apenas existen diferencias entre los conceptos y las relaciones conceptuales utilizadas en los diferentes niveles educativos, que, de hecho, se corresponden a momentos distintos en el desarrollo cognitivo del alumno. El mismo comentario tenemos que hacer con respecto a las explicaciones. En un rastreo asistemático de los libros de texto hemos encontrado una semejanza casi total en las explicaciones en todos los cursos. En todo caso, las diferencias encontradas no se debían al nivel educativo, sino a las diferencias en la óptica disciplinar de los autores de los libros (por ejemplo, los autores más inspirados en posiciones marxistas recurrian exclusivamente a explicaciones causales utilizando factores socioeconómicos; algunos autores más «humanistas" recurrian a explicaciones teleológicas o finalistas basadas en factores personales de los agentes históricos). En cualquier caso, en los libros de texto aparece habitualmente una confusión y sobre todo una superposición, nunca explícita, de ambos tipos de explicación. En este contexto, nuestra intención era estudiar los problemas que plantean a los alumnos algunos aspectos de la explicación en las ciencias sociales. Para nuestro estudio nos interesaba plantear una prueba que fuese una situación similar a las que habitualmente aparecen en las asignaturas de ciencias sociales. Del mismo modo los factores explicativos propuestos debían ser los que aparecen normalmente en sus libros de texto. Al mismo tiempo debería ser una prueba suficientemente significativa para las ciencias sociales y suficientemente abierta para que fuese accesible a los alumnos de cursos muy diferentes.

Por último debía ser una situación ficticia con el fin de que en las respuestas de los sujetos no recogiesen datos de la memoria específica, o episódica, de los alumnos. Con estos condicionamientos diseñamos la prueba que aparece más adelante.

\section{Objetivos e hipótesis}

Como hemos señalado anteriormente, nos interesaba comprobar cuáles eran los factores explicativos que se consideraban preferentemente a las distintas edades, así como cuáles eran las relaciones que aparecían entre esos factores distintos. A través de este estudio tratábamos de ver a qué edades los alumnos comienzan a utilizar de modo habitual un tipo de explicación 
que sea suficiente para comprender las situaciones sociales. $Y$ en su defecto qué tipo de comprensión presentan de estos temas.

Teniendo en cuenta las investigaciones anteriores conectadas con nuestro estudio y fundamentalmente las consideraciones expuestas más arriba teníamos en perspectiva las siguientes hipótesis de trabajo. En primer lugar esperábamos que los sujetos de diferentes edades recurriesen a distintos factores explicativos. Los más pequeños tenderían a considerar más importantes los factores más personalizados, que tengan que ver más con las personas y sus intereses. Los más mayores tenderían a considerar más importantes los factores más estructurales, que tienen que ver con la estructura social.

En segundo lugar esperábamos que los sujetos de distintas edades presentasen diferentes relaciones entre los factores explicativos. Los más pequeños establecerían escasas relaciones directas entre los factores y sus consecuencias. Los más mayores establecerían muchas relaciones entre los factores y sus consecuencias, y entre distintos factores, formando una compleja red de relaciones.

En tercer lugar, también esperábamos que los sujetos de distintas edades, cuando tuviesen en cuenta el mismo factor explicativo, lo hicieran por razones diferentes.

$\mathrm{Y}$ en cuarto lugar esperábamos que en torno a los quince-dieciseis años la mayoría de los sujetos alcanzasen un nivel mínimo de explicación, que les permitiera encarar con éxito las distintas situaciones de las ciencias sociales.

\section{Sujetos}

Para esta tarea seleccionamos una muestra de sujetos de EGB y BUP, pertenecientes a un co- legio y un instituto de las afueras de Madrid ${ }^{8}$. Su nivel socioeconómico podemos calificarlo de medio-bajo.

Se escogieron grupos de niños de los siguientes niveles: 10 alumnos de $6^{\circ} \mathrm{EGB} ; 20$ de $8^{\circ}$ de EGB; 20 de $2^{\circ}$ de BUP; 20 de COU de ciencias, y 20 de COU de letras. En total eran 90 sujetos. Los alumnos de COU estaban seleccionados, de acuerdo con la clásica división entre ciencias y letras, con optativas diferentes. Los sujetos fueron seleccionados al azar (orden de lista, por apellidos) y fueron eliminados aquellos que de forma sistemática obtenían calificaciones muy buenas o muy malas en las asignaturas de ciencias sociales. Todos los alumnos tenían la edad media de cada curso y eran voluntarios.

\section{Procedimiento}

Antes de la aplicación definitiva de la prueba a los sujetos que acabamos de indicar se realizó una prueba piloto con un reducido grupo de sujetos por nivel (cinco) con el fin de poner de manifiesto los problemas de la situación experimental propuesta y de obtener a grandes rasgos las respuestas de los sujetos. A partir de dicha prueba se modificó levemente la situación experimental y se elaboraron las categorías fundamentales de respuesta, que se comentarán más adelante. La tarea finalmente utilizada era la siguiente. Se proponía a los sujetos que resolvieran el siguiente problema: «Vas a leer estas tarjetas, en ellas se habla de un país en el que han ocurrido una serie de cosas. No es un problema que se pueda hacer bien o mal, solamente tienes que pensar sobre ello y darnos tu opinión, lo que tú pienses.»

En primer lugar se le daba a leer el texto general que aparece a continuación:

\section{La prosperidad de Tasmania}

"En Tasmania, un pequeño país del interior del continente, se babia producido un importante avance en los últimos años. Todo el país babia mejorado y la gente vivía mucho mejor que antes. Se trataba de un verdadero salto bacia adelante en todos los sectores. Los niveles económicos babian subido basta alcanzar los de los países más desarrollados, el nivel cultural de todo el país era cada vez más alto y abundaban las actividades artísticas."

"Todo el mundo se pregunta por qué ha ocurrido este progreso. Los observadores de otros paises se plantean cuál o cuáles pueden ser las razones de este avance tan espectacular de Tasmania.»

"A continuación se presenta una serie de tarjetas que explican algunos hechos ocurridos en Tasmania. La tarea consiste en que nos comentes si estos bechos pueden o no explicar el progreso y 
Se comentaba cualquier duda que plantearan los sujetos. Ante las solicitudes de más información, se anotaban y no se ofrecía nueva información, se repetía el texto o se posponía la duda para después de las tarjetas.
En segundo lugar se proporcionaban las siete tarjetas. El sujeto leía cada tarjeta y daba su opinión sobre la influencia o no de dicho suceso en la prosperidad del país. Concretamente, se le. pregunataba en cada tarjeta si influía, o no, y por qué. Las tarjetas eran las siguientes:

\section{TARJETA 1.}

"Hacía casi 150 años, el país no se babía mezclado en las guerras de la zona, habiendo tenido un desarrollo pacifico en su vida cotidiana.»

\section{TARJETA 2.}

"Se produjo un cambio en el clima, que pasó a ser más benigno, lo cual mejoró sensiblemente las cosechas y posibilitó la ganadería."

\section{TARJETA 3.}

"Desde que se recordaba, el país no babia sido especialmente próspero, y habia mantenido un nivel bajo de desarrollo, por lo que la gente pensaba que abora babía llegado la bora de la prosperidad para su pueblo."

\section{TARJETA 4.}

"El descubrimiento de nuevas técnicas de extracción babia posibilitado la explotación de los yacimientos mineros del sur del país."

\section{TARJETA 5.}

"La mayoría de la población se babia convertido a una nueva religión, que prometía la felicidad y la prosperidad para todos sus seguidores.»

\section{TARJETA 6.}

"Habian proliferado por el país unos grupos de estudiosos que organizaban reuniones de difusión cientifica, filosófica, artística y politica; algunos de estos miembros formaban parte del gobierno $y$ de los mandos de la administración."

Habitualmente, los sujetos solicitaban más información del experimentador. Ante esto, se le proponia razonar sobre la información que tenía delante y dejar para el final la consideración de nueva información. Cuando ya había opinado sobre las diferentes tarjetas y había dado una explicación global, se le proponía un diálogo más abierto, donde era posible considerar diferentes opciones.

Con todas las tarjetas sobre la mesa se pedía una explicación global de la prosperidad del país. También se le pedía que ordenase las tarjetas: las que no influyen, las que sí influyen, y en caso de que unas influyeran más y otras menos se le pedía que las ordenase. En esta fase se utilizaban normalmente las afirmaciones que el sujeto había hecho anteriormente. A continuación se le proponía al sujeto que pensase qué habría ocurrido si no se hubiera dado la tarjeta que él consideraba más importante y sí todas las demás. Se repetía con la segunda tarjeta en importancia. Se le pedía su opinión sobre lo que habría pasado respecto a la prosperidad del país. Por último se planteaba al sujeto, sobre sus afirmaciones anteriores, cuáles eran las relaciones entre las diferentes facetas del desarrollo de un país económico, artístico, político, etc.

A todos los efectos los factores o ideas explicativas recogidas en las tarjetas podrán ser identificadas por el número de cada tarjeta. Así cuando hablemos del factor 5 estamos haciendo referencia a la tarjeta número 5 , es decir, la religión.

La prueba fue administrada individualmente siguiendo el método clínico (Piaget, 1926). Se registró magnetofónicamente para facilitar el análisis. También se tomó un registro escrito.

\section{Criterios de análisis y resultados}

La corrección de la prueba tiene dos partes diferentes. Por un lado nos interesaba analizar las ideas espontáneas sobre los factores propuestos (guerra, clima, desarrollo temporal, nuevas técnicas, religión, inversión extranjera, y grupos de presión). Queríamos saber cuáles eran consideradas relevantes e irrelevantes. Por otra parte nos interesaba comprobar cuál era el tipo de re- 
lación que se establecía entre los distintos factores explicativos, independientemente de su contenido. A continuación se expondrán por separado ambos aspectos de la prueba, que no obstante constituyen un todo indivisible. Dada la complejidad de los criterios de análisis se detallarán conjuntamente con los resultados obtenidos en cada caso, para hacer más fácil su lectura.

\section{PARTE I}

Como comentábamos en los objetivos de la investigación, nos interesaba comprobar si los sujetos más pequeños y los mayores diferían en cuanto a la consideración de los diferentes hechos históricos planteados, como factores explicativos. $A$ priori, nuestra hipótesis era que los sujetos más pequeños darían una mayor importancia a los factores más personalizados, mientras que los mayores tenderían a considerar en mayor medida los factores o ideas explicativas más estructurales, aquellos factores que aludían a una situación más profunda, más compleja, más representativa de todo el funcionamiento social. En nuestra tarea cada tarjeta recogía un hecho que podía considerarse como un factor o idea explicativa interviniente; así, por ejemplo, pensábamos que en nuestra prueba los sujetos más pequeños elegirían las tarjetas con factores causales más personalizados, como la guerra (tarjeta 1), el grupo de presión (tarjeta 7), la religión (tarjeta 5). Por contra, los sujetos mayores elegirían los factores más estructurales, tales como la inversión extranjera (tarjeta 6), el aumento de la producción minera (tarjeta 4) o agrícola y ganadera (tarjeta 2).

A partir de estas consideraciones nos planteamos un primer nivel de análisis donde se pusiera de manifiesto la importancia de los distintos factores explicativos para los sujetos de los diferentes grupos de edad. Recordemos que a cada alumno se le pedía que ordenara, según su influencia, las distintas tarjetas. Los sujetos primero ordenaban de una parte las tarjetas que influyen y de otra las que no influyen. Después se les pedía que ordenaran de mayor a menor influencia este segundo grupo (como comentaremos más adelante los sujetos más pequeños curiosamente también ordenan las que no influyen). En muchas ocasiones los sujetos consideraban que dos o más factores ocupaban el mismo lugar de influencia. Se recogía su opinión y se tenía en cuenta posteriormente. Veamos un par de ejemplos: Un alumno de COU creía que «la 5 y la 3 no influyen nada, y de las otras la más importante es la 1 , luego la 2,4 y 6 son igualmente importantes, y la que menos, la 7 ». Y uno de $8^{\circ}$ de EGB decía: «Esto va de mucho a poco; así: 7, 1, 6, 2, 4, 5 y 3.» Es importante observar el tipo de estructura que presentan estas dos respuestas, al que volveremos más adelante.

Estos órdenes propuestos por los sujetos se ponderaban para elaborar una tabla que aparece a continuación como Cuadro I. En él aparecen en vertical los niveles educativos estudiados y en horizontal un orden de primero a séptimo (por ser siete las tarjetas propuestas en la tarea). Dentro del cuadro se ven unos números del 1 al 7 que aluden al número de las tarjetas según aparecen en la prueba (1-guerra, 2-clima, 3-tiempo, 4-técnicos, 5-religión, 6-inversión, 7-grupos). Por último, entre paréntesis aparece un coeficiente que expresa el promedio del lugar que ocupa esa tarjeta para los sujetos de ese nivel educativo. Este promedio viene dado por todos los sujetos de cada curso, en función del lugar en que cada uno sitúa a ese factor en su jerarquía explicativa. La ponderación era de 1 a 8 en función de que el factor ocupase del primero al séptimo lugar en la jerarquía del sujeto, y el 8 cuando afirmaba que un factor no influye nada. Veamos los dos sujetos anteriormente comentados: el alumno de 8 citado anteriormente recibiría un 1 para la tarjeta 7 , un 2 para la 1,3 para la a 6,4 para la 2,5 para la 4,6 para la 5 y 7 para la 3. El alumno de COU citado también anteriormente recibiría un 1 para la tarjeta 1 , un 2 para las tarjetas 2,4 y 6 , un 5 para la tarjeta 7 y un 8 para las tarjetas 3 y 5 . De esta forma un factor que todos los sujetos considerasen que no influye aparecería en la tabla con un ocho entre paréntesis. Un factor que todos colocasen como más importante figuraría en la tabla con un uno entre paréntesis.

En resumen, en la tabla aparece el orden de los factores explicativos para cada nivel educativo, que corresponde a la media de todo el grupo. Por ejemplo, en $6^{\circ}$ de EGB, el orden ideal de los factores es $6-7-1-2-4-3-5$ y entre paréntesis aparece el promedio del grupo. Esto quiere decir que la tarjeta 6 es la que ha sido colocada más veces como más importante, con un promedio ponderado de lugar de 4,1.

Si se analizan con detalle, los resultados que se recogen en el Cuadro I son suficientemente claros con respecto a las cuestiones planteadas anteriormente. En primer lugar cabe destacar que no se cumple nuestra expectativa de que los sujetos de los diferentes niveles mostrasen preferencia por diferentes factores explicativos. Por contra, en el cuadro podemos ver que los sujetos de los diferentes niveles presentan órdenes de importancia parecidos. Las diferencias encontradas entre las distintas tarjetas o factores explicativos se mantienen de unos niveles a otros. En 


\begin{tabular}{|l|l|l|ll|l|l|l|}
\hline & Primero & Segundo & Tercero & Cuarto & Quinto & Sexto & Séptimo \\
\hline 6.9 EGB & $6(4,1)$ & $7(4,4)$ & $1(4,6)$ & $2(4,6)$ & $4(4,6)$ & $3(5,7)$ & $5(5,8)$ \\
8.9 EGB & $4(3,0)$ & $2(3,1)$ & $1(3,6)$ & $6(3,8)$ & $7(4,2)$ & $3(6,4)$ & $5(6,9)$ \\
2.9 BUP & $1(2,2)$ & $4(2,8)$ & $2(3,3)$ & $6(3,4)$ & $7(3,9)$ & $3(6,8)$ & $5(7,8)$ \\
COU C & $7(2,7)$ & $2(3,0)$ & $1(3,1)$ & $4(3,2)$ & $6(4,2)$ & $5(6,5)$ & $3(7,4)$ \\
COU L & $4(2,7)$ & $7(3,1)$ & $1(3,3)$ & $6(3,5)$ & $2(3,7)$ & $3(7,0)$ & $5(7,3)$ \\
\hline
\end{tabular}

todos los niveles educativos (salvo $6^{\circ}$ de EGB) los factores 3 y 5 están siempre en los últimos lugares (sexto y séptimo), lo cual da una idea del escaso valor que conceden los sujetos a estos factores a la hora de explicar el hecho propuesto. El resto de los factores oscilan, todos ellos, entre 2,2 y 4,2 , sin que se pueda observar una tendencia clara en el lugar que ocupan, ni en el valor promedio, de unos sobre otros.

A la vista de estos datos podemos sostener que los sujetos de distintos niveles educativos, y por tanto, de diferentes edades, presentan la misma tendencia a considerar unos factores explicativos u otros. No obstante, esta similitud, al menos en el caso de $6^{0}$ de EGB, es sólo aparente. Como recoge el Cuadro I, los alumnos de $6^{\circ}$ de EGB, a diferencia de los mayores, apenas muestran preferencia por unos factores con respecto a otros. Pero si los factores utilizados son muy semejantes en los distintos cursos no sucede lo mismo con las explicaciones elaboradas a partir de ellos. Un mismo factor puede considerarse importante por razones muy diferentes y hasta opuestas. Otra de nuestras previsiones se refería a que los sujetos de los diferentes grupos de edad otorgarían un peso diferente a los distintos factores, es decir, que, aunque los factores considerados sean los mismos, las explicaciones elaboradas a partir de ellos serán diferentes.

En los protocolos de los sujetos apareció claramente esta diferencia. Los sujetos más jóvenes establecen una explicación de la influencia de los diferentes factores que se reflejan en relaciones directas y simples, mientras que los más mayores integran los factores en una explicación mucho más dinámica. Nuestra situación experimental no se presta especialmente a realizar un análisis exhaustivo sobre esta cuestión, y por lo tanto sería necesario investigar más este punto con otras situaciones experimentales. Sin embargo, estas diferencias encontradas están en la misma línea que los datos encontrados en numerosas investigaciones sobre la explicación causal, comentadas anteriormente (por ej., Pozo 1985b). A partir de ellas y de nuestros propios datos po- demos mantener que los sujetos más pequeños difieren sensiblemente de los mayores en la consideración que hacen de los hechos históricos y en las explicaciones que elaboran.

Existe otra consideración importante respecto a los diferentes factores explicativos propuestos. Se trata de su relevancia desde el punto de vista disciplinar. Si bien todos estos factores han sido recogidos de textos realizados por historiadores, parece claro que no todos compartirían la misma importancia para un grupo representativo de historiadores. Es decir, desde el punto de vista de los expertos no todos estos factores presentados gozarían del mismo poder explicativo. Bien es cierto que podríamos encontrar entre expertos de distintas corrientes historiográficas argumentos a favor de todos ellos, pero en algunos casos sería más difícil encontrar estos argumentos. Era esperable que en los resultados también se reflejase esta situación.

En el Cuadro I encontramos algunos datos que apoyan esta interpretación. En todos los niveles educativo, existe una marcada diferencia entre la consideración de unos factores explicativos y otros. Podemos ver que las tarjetas 3 y 5 prácticamente no son consideradas por los sujetos, mientras que las otras tarjetas tienden a ocupar siempre mejores lugares. Asimismo, en los protocolos de los sujetos se observa una mayor relación entre estos últimos factores mientras que el 3 y el 5 son considerados siempre de forma aislada. No obstante, como ocurría en el comentario anterior, para realizar un análisis detallado de esta cuestión sería preciso recurrir a otras pruebas que recojan especialmente este aspecto.

En resumen, estas últimas consideraciones nos hacen ver la importancia de las relaciones entre unos factores y otros, que pasamos a estudiar en la segunda parte de la investigación.

\section{PARTE II}

Vamos a describir los niveles de relación encontrados en las respuestas de los sujetos. A partir de la prueba piloto y del análisis formal de 
la tarea se elaboraron cuatro niveles de distinta complejidad entre las relaciones de los hechos propuestos. Los niveles que aparecen a continuación son inclusivos, de modo que cada nivel no sólo implica más elaboración que el anterior, sino que además se apoya en las adquisiciones precedentes.

El primer nivel, expresado fundamentalmente por la idea de que no existe la necesidad de explicación, generalmente unido a una visión estática de la realidad social. En este nivel los sujetos no sienten la necesidad de explicar que un pais sea rico o pobre porque «todo el mundo sabe que hay países ricos y países pobres». Los sujetos no son consistentes en sus explicaciones, pueden sostener que un factor influye a favor de que un país se desarrolle y a continuación sostener que no influye o que influye en contra, sin ningún tipo de explicación. En una tarjeta pueden decir que algo influye y negarlo en la siguiente. Son aquellos sujetos que repiten las tarjetas de forma sistemática como argumento de su influencia: «Sí, porque hubo un cambio en el clima que mejoró las cosechas.» (Y ¿por qué?) «Porque hubo un cambio en el clima que mejoró las cosechas.» Es curioso y muy significativo observar cómo estos sujetos ordenan los factores causales que según ellos no influyen en la situación: «Estos tres no influyen, pero éste menos que éste, y éste menos que el otro.» (Entonces, ¿influyen un poco?) «No, ninguno de éstos influye nada, pero éstos menos que éste.» En términos generales este nivel se corresponde con el estadio «descriptivo» hallado en otras investigaciones anteriores sobre el desarrollo de las explicaciones en Ciencias Sociales (por ejemplo: Peel, 1971; Pozo y Carretero, 1983).

El segundo nivel viene expresado fundamentalmente por la comprensión de las ideas causales como un hecho aislado que provoca un efecto con el que no existe de manera explícita una relación precisa. Los sujetos de este nivel generalmente opinan que un hecho provoca, o no, un efecto o consecuencia, pero no explican en ningún momento cómo se produce esa influencia o por qué se produce. Estos sujetos no engloban el factor causal en una cadena de causas y efectos que provocan un resultado final. De esta forma la consecuencia última, que en nuestro caso es lo que hay que explicar (la prosperidad del país), sigue siendo una consecuencia mágica (por no explicada) de la identificación de los factores influyentes. Por ejemplo, nos pueden comentar: «La guerra siempre es mala para un país.» ( $¿ I n-$ fluye?) «Sí, porque la guerra es mala y perjudica al país»; «que otros les den dinero siempre es bueno, porque así podrán hacer más cosas e irán mejor.»
El tercer nivel viene expresado fundamentalmente por la aparición de una concepción causal precisa. En este nivel es donde podemos empezar a hablar de que el sujeto tiene una cierta concepción de lo que es la causalidad. El sujeto de este nivel es capaz de establecer cadenas causales, más o menos extensas y precisas, sobre la causa y los sucesivos efectos que a su vez son causas de otros efectos. Los sujetos que se sitúan en este nivel forman un sistema de concatenaciones causa-efecto/causa-efecto/causa-efecto..., que provocan un resultado final, en nuestro caso el desarrollo del país. Nos dirán: «El clima mejoró las cosechas, al tener más cosechas pueden vender más, y se lo pueden vender a otros países, y con ese dinero pueden mejorar el país»; «la guerra es siempre mala porque provoca destrucción de las casas y de las fábricas, y muere mucha gente, al estar las fábricas destruidas y sin gente no se puede producir nada y el país se empobrece»; «la inversión extranjera permite realizar proyectos previstos, como fábricas, así se crean puestos de trabajo, y la gente que puede trabajar está más contenta y trabaja con más ganas, y eso hace que el país produzca más y se desarrolle».

El cuarto nivel viene expresado fundamentalmente por la aparición de interacciones causales propiamente dichas. Los sujetos son capaces de establecer cadenas causales sistemáticas y poner en relación unas causas con otras. $\mathrm{Y}$ también pueden establecer uniones entre unas cadenas causales y otras, y darse cuenta de que un factor puede influir a favor y en contra al mismo tiempo. Por último comprenden también que un factor puede influir de modo diferente en función de la relación con los otros factores y de la situación a la que se aplique. Son los sujetos que nos dicen: «La inversión extranjera puede ser beneficiosa a corto plazo porque mete dinero en el país y eso permitirá crear fábricas y trabajo, pero será perjudicial a medio plazo porque habrá que devolver el dinero a quien lo haya prestado y con intereses»; «la guerra puede influir negativamente si el país i iterviene de modo directo, pero si la guerra es fuera y ellos sólo les venden las armas puede ser muy beneficiosa»; «un cambio en el clima puede influir, pero depende del tipo de economía que tenga el país; si es una economía muy primaria influirá más que si es una économía basada en la industria».

En este nivel cuatro vemos que aparece un verdadero modelo de las relaciones entre los hechos, que permite una explicación global. Es en este nivel cuando los sujetos son capaces de anticipar factores explicativos y de ofrecer explicaciones acabadas antes de tener presentes todas las tarjetas. Con posterioridad reordenar el conjunto a la vista de nueva información relevante. 
En este sentido resultan clarificadoras las preguntas que se hacían al sujeto de modo sistemático al final de la prueba. Recordemos que cuando el sujeto había acabado de ordenar todas las tarjetas propuestas se le preguntaba qué habría pasado si el factor que él consideraba más importante no se hubiese producido. Después se repetía la pregunta con el. segundo factor en importancia. Ante la pregunta, la práctica totalidad de los sujetos (salvo los del nivel IV) o bien no la entendían o bien la consideraban de un modo aditivo. Los sujetos más pequeños contestaban de forma inconsistente o no entendían. Son los que nos decían por ejemplo: «no sé, porque sí se produjo». La mayoría de los sujetos presentaban una relación aditiva, es decir, consideraban que se había producido el mismo efecto pero atenuado por la ausencia de uno de los factores principales. Por ejemplo: «Se había dado igual, pero un poco menos, y con el segundo igual, lo mismo pero algo menos." Por el contrario, en el nivel IV nos encontramos con una concepción dinámica e integrada que ofrece una relación multiplicativa totalmente distinta de la anterior. Por ejemplo: «Si no se produce éste no sé lo que hubiese ocurrido, porque todos se influyen unos en otros, y uno solo no vale para explicarlo todo, al faltar éste lo mismo no se hubiese producido ninguna prosperidad, o lo mismo se hubiese producido la misma, depende de todo lo demás.»

Cabría realizar aquí algunas apreciaciones. En primer lugar, hay que reconocer que el tipo de relación causal exigido en el nivel cuatro y sobre todo en el nivel tres es una relación todavía simple si se compara con las relaciones establecidas por los expertos, por los historiadores. Sin embargo se puede reconocer que nos movemos ya en unos niveles de explicación relativamente aceptables. En segundo lugar, hay que aclarar que las respuestas de los sujetos no eran las mismas para unos factores causales que para otros. A la hora de considerar a los sujetos en un nivel u otro se tuvo en cuenta la consistencia de todo el protocolo. En general, los sujetos fueron bastante coherentes respecto al nivel urilizado, ya que la mayoría de las respuestas pertenecian al mismo nivel aunque versaran sobre factores distintos. En otro lugar realizamos un análisis de las diferentes respuestas a unos factores y otros $(\mathrm{Ca}$ rretero, Asensio y Pozo, 1985).

A continuación aparece el Cuadro II donde se reflejan los resultados obtenidos por los sujetos respecto a los diferentes niveles de edad. Las respuestas vienen categorizadas en función de los niveles que acabamos de describir.

\section{CuAdro II}

Niveles de relación entre los factores explicativos en cada curso*

\begin{tabular}{|l|c|c|c|c|}
\hline & I & II & III & IV \\
\hline 6.9 EGB & $1(10 \%)$ & $9(90)$ & - & - \\
8.9 EGB & $3(15)$ & $17(85)$ & - & - \\
2.9 BUP & - & $11(55)$ & $9(45)$ & - \\
COU C & - & - & $14(70)$ & $6(30)$ \\
COU L & - & - & $4(20)$ & $16(80)$ \\
\hline
\end{tabular}

* Chi-Cuadrado significativo más allá del nivel 0,001, Chi-Cuadrado $=97,8 ; \mathrm{p}<0,001$.

Pasemos a comentar el Cuadro II. Se confirma que los sujetos de diferentes grupos educativos establecen diferentes tipos de relaciones entre los factores explicativos ${ }^{9}$. Se afirma así que los sujetos más pequeños no establecen relaciones entre los factores, mientras que sí lo hacen los mayores, estableciendo un tipo de relación más dinámica y compleja. Tan sólo un 50 por 100 de los alumnos de $2^{\circ}$ de BUP se encuentran en el nivel tres, que es en el que podemos hablar ya de un nivel aceptable de explicación, y el otro 50 por 100 sigue en el nivel dos, es decir, dando pseudo-explicaciones, sin ningún tipo de relación causal o teleológica concreta entre los hechos propuestos y la prosperidad del país. Podemos observar que el 85 por 100 de los sujetos de $8^{\circ}$ de EGB se sitúan en el nivel dos. Este dato nos pone de manifiesto que el tipo de relación entre los factores explicativos que el alumno es capaz de comprender y utilizar contrasta con el nivel de complejidad de las explicaciones a que se ve enfrentado de modo continuado en el aula.

En general, hay que esperar a COU para que todos los sujetos se sitúen en los niveles tres y cuatro. Recordemos una vez más que tan sólo en estos niveles podemos hablar con propiedad de explicaciones. En $2^{2}$ de BUP todavía la mitad de los alumnos no entran en estos niveles.

En nuestros datos hay otros dos resultados que nos parecen relevantes. En primer lugar, el es- 
caso número de sujetos en el nivel uno, incluso en los niveles educativos más bajos. Y por otra parte, la diferencia entre el COU de ciencias y el de letras. El hecho de que haya tan pocos sujetos en el nivel uno quiere decir que la mayor parte de éstos, por pequeños y poco instruidos que estén, comprenden la necesidad lógica de que exista una relación entre los antecedentes y los consecuentes de una situación. Este dato no es sorprendente en absoluto. Existen otros muchos dominios donde estas relaciones se establecen mucho antes de la adolescencia. De esta manera los alumnos tratan de explicarse la realidad a partir del convencimiento de que existe una determinada relación entre los eventos que se producen y que unos hechos son determinantes de los otros, aunque desconozcan su existencia, sus relaciones, y hasta su funcionamiento. En varias ocasiones hemos comentado la necesidad de partir de los conocimientos y capacidades de los alumnos. Estos datos son un apoyo más a favor de que el alumno no es una tabla rasa donde escribir los conocimientos históricos. Cada alumno dispone de un bagaje previo de opiniones y actitudes que va a condicionar cualquier conocimiento posterior que deba asimilar. Estos datos enlazan con un gran número de investigaciones (realizadas en su mayoría sobre nociones de las ciencias naturales) que han puesto de manifiesto la importancia de las ideas previas - muchas veces erróneas - y la importancia de ponerlas de manifiesto como requisito previo para el aprendiźaje (por ejemplo: Carretero, 1985; Pozo, 1985b). A este respecto sería necesario una mayor investigación en estas cuestiones para el caso de las ciencias sociales. Parece que en éstas las concepciones erróneas son menos consistentes y las ideas previas menos abundantes. Nuestros sujetos demostraban más una ausencia de conocimiento que unas ideas previas consolidadas.

Respecto a los resultados de los grupos de COU, puede resultar sorprendente que exista tanta diferencia entre ambos grupos, ciencias y letras. Es necesario puntualizar que nuestros datos se recogieron al final del curso, $y$, por tanto, los alumnos llevaban dado prácticamente todo el programa de cada asignatura. En primer lugar habría que decir que, para generalizar estos resultados, sería necesario realizar una investigación más específica sobre esta variable en concreto. Sin embargo, si tenemos en cuenta la investigación psicológica más reciente estos datos no son sorprendentes en absoluto. Podríamos decir que a partir de los trabajos sobre las diferencias entre expertos y novatos (por ejemplo: Chi, Glaser y Rees, 1982; Voss, Tyler y Yengo, 1983), los resultados de nuestra investigación son los esperables. La diferencia fundamental entre ambos grupos de $\mathrm{COU}$ es que los alumnos de letras han aprendido una serie de «modelos explicativos» o «teorías» que pueden aplicar a la nueva situación y que les ayuda a comprenderla. Por su parte es esperable que con una tarea de tipo físico los datos obtenidos hubiesen sido a la inversa, como hemos comprobado en una investigación recientemente realizada por uno de nostros (Pozo, 1985b).

\section{CONCLUSIONES}

Hemos visto en nuestro estudio que la explicación histórica plantea muchos problemas, tanto en sus aspectos disciplinares y epistemológicos como en su vertiente psicológica. La explicación correcta de los hechos históricos excede en muchos casos las capacidades cognitivas de los alumnos. En nuestro trabajo se puede observar que muy pocos sujetos llegan a poseer un nivel de comprensión suficientemente elaborado como para encarar con éxito las explicaciones en ciencias sociales. Hay que esperar a COU para que un número aceptable de alumnos posea este nivel y aún en este curso hay muchos sujetos que no lo alcanzan. Pero, al tiempo, en el aula se está sometiendo a los alumnos a un tipo de explicaciones muy alejadas de sus propias concepciones y que, por tanto, no son capaces de entender. Estos alumnos, como irónicamente nos decía uno de ellos, tendrán que memorizar los hechos y además las explicaciones de los mismos.

Pero estos datos no deben ser interpretados con pesimismo. En ellos aparece una gran influencia de la instrucción que reciben los alumnos, que en última instancia se torna decisiva. La mayor parte de las diferencias entre los alumnos de ciencias y de letras se debe explicar en función del aprendizaje realizado en el aula. Parece lícito pensar que en estos alumnos han adquirido una serie de «modelos explicativos» que les permiten comprender mejor situaciones concretas a las que poder aplicarlos. En este sentido sería conveniente orientar el aprendizaje hacia la provisión de estos modelos, que constituyan un verdadero bagaje de conocimiento de la realidad.

Habría que diferenciar en un primer nivel los conceptos fundamentales de la disciplina, en este caso de las ciencias sociales, aquellos «verdaderos ladrillos del conoci- 
miento humano». En un segundo nivel, se situarían los «modelos explicativos», que se aplicarían a partes más amplias de la realidad, y que servirian para explicar situaciones sociales más complejas, de modo que establezcan determinadas relaciones entre los conceptos. Por último, en un tercer nivel, se situaría una determinada concepción disciplinar o ideológica, mucho más global, que representaría una óptica específica para ana lizar los problemas sociales. Desde este tercer nivel se primarán unos determinados modelos explicativos sobre otros. Las relaciones entre los tres niveles son complejas, pero desde un punto de vista psicopedagógico esta diferenciación puede ayudar a clarificar los problemas por los que atraviesa la comprensión del alumno. Posiblemente ese tercer nivel sólo resulte accesible a los propios historiadores o profesionales de la historia y habría que conformarse con que los alumnos adolescentes accedan a los dos anteriores.

Es claro que para que el alumno llegue a poseer un cierto modelo del funcionamiento y cambio de las sociedades la enseñanza que actualmente se les transmite es insuficiente. Para que el alumno elabore ese modelo es necesario que tenga un papel más activo en la construcción de su propio conocimiento. Afortunadamente, esta idea está siendo asumida ya por muchos profesores e incluso constituye ya uno de los lemas «oficiales» del cambio educativo en este área. Por ello, no es necesario abundar más en su necesidad. Más bien, parece necesario advertir de los peligros que comporta su aplicación irreflexiva o excesivamente apresurada al ámbito escolar.

Los proyectos renovadores en la enseñanza de las ciencias sociales, basados en su mayor parte en la idea constructivista, tienden a destacar las actividades de descubrimiento por parte del alumno. Así, por ejemplo, el más prestigioso de ellos, el proyecto $H$ istory 13-16, parcialmente adoptado al castellano (Varios, 1985), insiste en la necesidad de que el alumno domine las técnicas y recursos metódicos del historiador. Se trata de que el alumno haga historia en vez de recibirla ya hecha. Para ello sin duda es necesario que domine todos esos conceptos $\mathrm{e}$ instrumentos metódicos que el Proyecto se propone transmitir. Esos conceptos están, desde un punto de vista psicológico, ligados al desarrollo del pensamiento científico o formal. Pero, como hemos indicado anteriormente, tanto los estudios psicológicos (véase Carretero, 1985; Pozo y Carretero, 1986) como didácticos (Ausubel, Novak y Hamesian; 1978), e incluso epistemológicos (por ejemplo, Lakatos, 1978), muestran que la elaboración del conocimiento científico, y por tanto su progreso o desarrollo, no está ligado sólo al desarrollo de nuevas formas de pensamiento sino sobre todo del dominio de nuevos sistemas o modelos conceptuales que permitan abordar de una nueva forma los problemas. En otras palabras, el desarrollo del pensamiento formal, en el que están y deben estar empeñados todos los intentos de renovación, es una condición necesaria pero no suficiente para acceder al conocimiento y la explicación histórica. Se requiere además poseer un modelo explicativo (sea causal, intencional o una integración de ambos) que el pensamiento formal no necesariamente asegura, ya que depende. del conocimiento de mecanismos causales o intencionales con un contenido propio. Del mismo modo que el pensamiento formal no asegura la comprensión de las leyes de Newton (¿cuántos profesores de historia -que supuestamente piensan de un modo formal- comprenden esas leyes?), tampoco asegura la comprensión del funcionamiento social. Unicamente lo hace posible. $Y$ para que esa posibilidad se convierta en realidad es necesario transmitir al alumno redes conceptuales que faciliten esa comprensión. La delimitación de esas redes conceptuales constituye un problema de enorme envergadura que ya ha sido emprendido en otras áreas de las ciencias sociales - por ejemplo, en geografía disponemos de los excelentes trabajos de Capel y colaboradores (véase Capel, Luis y Urteaga, 1984) - , pero no en el ámbito de la historia.

Es ésta una tarea de clasificación urgente que no impide entretanto ir avanzando en la elaboración de modelos didácticos que incluyan la transmisión tanto de métodos de investigación y análisis como de sistemas o modelos de explicación de los hechos históricos. 
En el presente trabajo se analizan los requisitos cognitivos necesarios para alcanzar los dos tipos de explicación que suelen admitirse en la bistoria y en las ciencias sociales: la causal y la intencional. Se estudian también las diferencias entre ambas y los sesgos propios de cada una de ellas. Se presenta, además, una investigación realizada con sujetos adolescentes en la que se muestran las dificultades para acceder a una explicación causal que integre los distintos aspectos de la realidad bistórica. Estas dificultades no se superan hasta los diecisiete-dieciocho años $y$ no en todos los casos.

\section{Summary}

In this paper cognitive demands in order to understand causal and intentional explanation in bistory and social sciencies are analyzed. Differences between these two kinds of explanations and their caracteristic biases are also studied.

A number of adolescents subjects were examined. Their difficulties to formulate a causal explanation in order to integrate different bistorical aspects are analyzed. These difficulties are overcome by the age of 17-18 years but only in some cases.

\section{Résumé}

Le présent article passe en revue les conditions necessaires pour la réalisation des explications bistoriques. Ou y examine l'explication bien causale qu'intentionelle, en signalant les biaises les plus fréquents. On expose en plus une recherche réalisée avec des adolescents où on montre les difficultés des adolescents pour intégrer des diverses niveaux de la realité dans l'explication bistorique. Cettes difficultés ne son pas surmontées que jusqu'à l'âge de 17-18 ans, et même pas dans tous les cas.

\section{Referencias}

ADELSON, J.: «The political imagination of the young adolescent» Daedalus, (4), (1971); 1013-1050.

ADELSON, J., Y O'NEIL, R.: «The growth of political ideas in adolescence: the sense of comunity". Journal of Personality and Social Psychology, 4(5), (1966); 295-406.

asensio, M.; Carretero, M., y POZo, J. I.: «La comprensión de la historia: pensamiento relativista». Cuadernos de Pedagogía; (1986); 133.

Ausubel, D. P.; Novak, J. D. y Hanesian, H.: Educational Psychology. A cognitive view. 2.a ed. Nueva York: Holt, Rinehart \& Winston, 1978. Trad. cast. de la 2. ${ }^{a}$ ed. de M. Sandoval: Psicologia Educativa. México: Trillas, 1983.

Biziére, J. M.: «Psicohistory and histoire des mentalités». The Journal of Psicobistory, 11 (1), (1983); 89-109.

BoARdman, D. (Ed.): New directions in geographical educations. Pasingstoke, The Palmer Ponss, 1985.

BooTH, M.: «A modern world history concepts and the thinking of adolescent pupils». Educational Review, 32 (3), (1980); 245-257.

BOPGER, R., y CIOFFI, F. (Eds.): Explanation in behavioural sciences. Cambridge, Cambridge University Press, 1970. Trad. cast. de J. D. Quesada: La explicación en las ciencias de la conducta. Madrid, Alianza, 1974.

Bullock, M.; Gelman, R. y Baillargeon, R.: «The development of causal reasoning, en W. J. Friedman (Ed.) The developmental psychology of time. Londres, Academic Press, 1982.

Bunge, M.: Causality. The place of the causal principle in modern science. Cambridge, Harvard University Press, 1959 (2.a ed., 1979). Trad. cast. de la 1. a ed. de H. Rodríguez: Causalidad. El principio de causalidad en la ciencia moderna. Buenos Aires, Eudeba, 1961 (4. ${ }^{a}$ ed., 1978).

- Racionalidad y realismo. Madrid, Alianza, 1985.

BuSs, A.: "Causes and reasons in atribution theory: a conceptual critique». Journal of Personality and Social Psycbology, 36, (1978); 1311-1321.

CAPEL, H.; LUIS, A. y URTEAGA, L.: «La geografía ante la Reforma Educativa». Geocritica, 1984, 53, completo.

CARR, E. H.: What is history? Londres, Pelican, 1961. Trad. cast. de J. Romero: ¿Qué es la bistoria? Barcelona, Seix-Barral, 1966.

CARretero, M.: «El desarrollo cognitivo en la adolescencia y la juventud: las operaciones formales», en: $M$. Carretero, J. Palacios y A. Marchesi (Eds.): Psicología Evolutiva. 3. Adolescencia, madurez y senectud. Madrid, Alianza, 1985.

Carretero, M. y Garcia Madruga, J. A. (Eds.): Lecturas de psicologia del pensamiento. Madrid, Alianza, 1984.

Carretero, M; Pozo, J. I. y AsEnsio, M.: «La comprensión de conceptos históricos durante la adolescencia». Infancia y Aprendizaje, 23, (1983); 55-74. 
Carretero, M.; Asensio, M. y Pozo, J. I.: «La comprensió de la história a $1^{\circ}$ EGB: una asignatura pendent». Perspectiva Escolar, 92, (1985a); 2-6.

- Ersteñana de la bistaria y desarrollo cognitivo (i i-16 atios). Bases psico-pedagógicas para la elaboración de un nuevo curriculum de Historia en la reforma de las Enseñanzas Medias. Memoria de investigación no publicada. Madrid, Instituto de Ciencias de la Educación de la Universidad Autónoma de Madrid, 1985b.

Collingwood, R. G.: The idea of bistory. Oxford, Clarendon, 1946. Trad. cast. de E. O'Gorman y J. Hernández: Idea de la bistoria. México, F.C.E., 1965 (2. ${ }^{\mathrm{a}} \mathrm{ed}$.).

CHI, M. T. H.; Glaser, R. y ReES, E.: «Expertise in problem solving», en R. Sternberg (Ed.): Advances in the psychology of buman inteligence, vol. 2. Hillsdale, N. J.: Erlbaum, 1982.

DICKINSON, A. K. y LeE, P. J. (Eds.): History teaching and bistorical understanding. Londres, Heineman, 1978.

Dickinson, A. K.; LeE, P. J. y Rogers, P. J.: Learning History. Liverpool, Hieneman Educational Books, 1984.

DOMINGUEZ, J.: «Enseñar a comprender el pasado: conceptos y empatía». Infancia y Aprendizaje, en este mismo número.

FisCHHOFF, B.: «For those condemned to study the past: heuristics and biases in hindsight», en, D. Kahneman, P. Slovic y A. Tversky (Eds.): Judgement under uncertainty. Cambridge, Cambridge University Press, 1982.

Graves, N. J. (Ed.): New Unesco Source Book for Geography Teaching. Hong Kong, Longman, The Unesco Press, 1982.

Hallam, R. N.: "Piaget and thinking in history", en M. Ballard (Ed.): New movements in the study and teaching of history. Londres, Indiane University Press, 1970.

KahNEMAN, D.; SLOVIC, P. y TVERSKY, A. (Eds.): Judgement under uncertainty: beuristics and biases. Cambridge, Cambridge University Press, 1982.

KELLEY, H. H.: «Attribution theory in social psychology», en D. Levien (Ed.): Nebraska Symposium on Motivation. vol. 15. Lincoln, University of Nebraska Press, 1967.

- «Causal schemata and the attribution process», en E.E. Jones et al.: Attribution: perceiving the causes of bebavior. Morristown, N. J., General Learning Press, 1972.

Kelley, H. H. y Michella, J. L.: «Attribution theory and research» Annual Review of Psychology, 31, (1980); 457-501.

LAKATOS, I. "The metbodology of scientific research programmes philosophical papers, volumen I (Ed. de J. Worall y G. Currie). Cambridge, Cambridge University Press, 1978. Trad. cast. de J. C. Zapatero: La metodología de los programas de investigación cientifica. Madrid, Alianza, 1983.

Luc, J. N.: L'bistoire par l'etude de milieu. París, Les Editions ESF. Trad. cast.: La enseñanza de la bistoria a través del medio. Madrid, Cincel-Kapelusz, 1981.

Manninen, J., y TuOmelA, R. (Eds.): Articles from "Explanation an understanding". Dordrecht, Reidel. Trad. cast. de L. Vega: Ensayos sobre explicación y comprensión. Madrid, Alianza, 1980.

MARTIN, E.: «Jugando a hacer historia: los juegos de simulación como recurso didáctico». Infancia y Aprendizaje, 24, (1983); 69-88.

NAISH, M. C.: "Mental development and the learning of the geography», en N. J. Graves (Edm.): New Unesco source book for geograpby teaching. Hong Kong, Longman/The Unesco Press, 1982.

NisBeTT, R., y Ross, L.: Human inference: strategies and shortcomings of social judgement. Englewood Cliffs, N. J., Prentice-Hall, 1980.

PeEL, F. A.: The nature of adolescent judgement. Londres, Staples Press, 1971.

PIAGET, J.: La representation du monde chez l'enfant. Neuchatel, Delachaux et Niestlé, 1926. Trad. cast. de V. Valls y Anglés: La representación del mundo en el niño. Madrid, Morata, 1973.

PIÑEIRO, M. R., y GIL, P.: «Los juegos de simulación en la EGB: una investigación en el área de las ciencias sociales». Infancia y Aprendizaje, 27/28, (1984); 185-204.

PLOWRIGHT, J.: «Watching the detectives: A critique of the Schools Council's Analogy between the Historian and the Detectives». Teaching History, 35, (1983); 6-9.

Pozo, J. I.: El niño y la historia. Madrid, M.E.C., 1985a.

- Teorias y reglas de inferencia en la solución de problemas causales. Tesis doctoral no publicada. Madrid, Universidad Autónoma de Madrid, 1985b.

Pozo, J. I., y CARretero, M.: «El adolescente como historiador». Infancia y Aprendizaje, 23, (1980); $75-90$.

- «¿Enseñar Historia o contar «historias»? Otro falso dilema». Cuadernos de Pedagogia, 111, (1984); $45-50$.

Pozo, J. I.; Carretero, M., y Asensio, M.: «Cómo enseñar el pasado para entender el presente. Observaciones sobre la didáctica de la historia». Infancia y Aprendizaje, 24, (1983); 55-68.

RIEGEl, K. F.: Psychology of development and bistory. Nueva York, Plenum Press, 1976.

- «Toward a dialectical interpretation of time and change». en B. S. Gorman y A. E. Wessman (Eds.): The personal experience of time. Nueva York, Plenum Press, 1977.

Toulmin, S.: «Causes and reasons», en R. Borger y F. Cioffi (Eds.): Explanation in behavioural sciences. Cambridge, Cambridge University Press, 1970. Trad. cast. de J. D. Quesada: La explicación en las ciencias de la conducta. Madrid, Alianza, 1974.

TVERSKY, A., y KAHNEMAN, D.: «Judgement under uncertainty: heuristics and biases». Science, 185, (1974); 1124-1131. Trad. cast. de J. I. Pozo en M. Carretero y J. A. García Madruga (Eds.): Lecturas de psicología del pensamiento. Madrid, Alianza, 1984.

Varios. Hacer Historia. Proyecto 13-16. Barcelona, CYMYX, 1985.

VEGA, M. de: Introducción a la psicología cognitiva. Madrid, Alianza, 1984.

VOSS, J. F.; TYLER, S. W. y YENGO, L. A.: "Individual differences in the solving of the social science problems», en R. F. Dillon y R. R. Schmeck (Eds.): Individual differences in cognition. Nueva York, Academic Press, 1983.

VON WRIGHT, G. H.: Explanation and understanding. Cornell, Cornell University Press, 1971. Trad. cast. de L. Vega: Explicación y comprensión. Madrid, Alianza, 1979.

ZacCaGnini, J. L.: «Modelos de mundo como marco de referencia de una psicología del conocimiento». Estudios de Psicologia, 22, (1985); 19-32. 